\title{
The lifespan of classical solutions to nonlinear wave equations in two space dimensions
}

\author{
Rentaro AgEmi and Hiroyuki TAKAMURA \\ (Received December 2, 1991)
}

\section{§ 1. Introduction}

In the present paper we study the lifespan of solutions to initial value problems for nonlinear wave equations of the form

$$
\begin{aligned}
& \partial_{t}^{2} u(x, t)-\Delta u(x, t)=A|u(x, t)|^{p},(x, t) \in \boldsymbol{R}^{n} \times[0, \infty), \\
& u(x, 0)=f(x), \partial_{t} u(x, 0)=g(x), x \in \boldsymbol{R}^{n},
\end{aligned}
$$

where $p$ and $A$ are positive constants and $n=2,3$.

F. John [6] has proved the following remarkable results in three space dimensions. The global classical solution to (1.1) exists for small initial data with compact support provided $p>p_{0}(3)=1+\sqrt{2}$, and the lifespan of classical solution to (1.1) is finite provided $1<p<p_{0}(3), f=0, g>0$ (also see F. John [7], p. 32). Here $p_{0}(n)$ stands for the positive root of the quadratic $q(n, p)=0$ where

$$
q(n, p)=\frac{n-1}{2} p^{2}-\frac{n+1}{2} p-1
$$

and the lifespan of a solution $u$ to (1.1) means the largest $T$ such that $u(x, t) \in C^{2}\left(\boldsymbol{R}^{n} \times[0, T)\right)$. He also proved in [6] that the lifespan $T_{\varepsilon}$ of solutions to (1.1) with $f(x)=\varepsilon \varphi(x)$ and $g(x)=\varepsilon \psi(x)$ is equivalent to $\varepsilon^{-2}$ for $p=2$. Recently H. Lindblad [9] has refined this result by showing that the following limit exists for $1<p<p_{0}(3)$ :

$$
\lim _{\varepsilon \rightarrow+0} \varepsilon^{-p(p-1) / q(3, p)} T_{\varepsilon} .
$$

R. T. Glassey [3] has proved in two space dimensions that the global solution to (1.1) exists for small initial data with compact support provided $p>p_{0}(2)$. R. T. Glassey [4] proved that if $1<p<p_{0}(2)$ then the lifespan of a solution to $(1,1)$ is finite. Moreover, J. Schaeffer [10] proved that the lifespan is finite for critical values $p=p_{0}(2)$ and $p_{0}(3)$.

The main aim of this paper is to look for the upper and lower bounds 
for the lifespan in two space dimensions.

THEOREM 1. Let $u_{\varepsilon}$ be a $C^{2}$-solution to (1. 1) with initial data $f(x)$ $=\varepsilon \varphi(x)$ and $g(x)=\varepsilon \psi(x)$, where $\varepsilon>0, \varphi \in C_{0}^{3}\left(\boldsymbol{R}^{2}\right)$ and $\phi \in C_{0}^{2}\left(\boldsymbol{R}^{2}\right)$. Then there exist positive constants $\varepsilon_{0}$ and $C$ depending only on $p, A, \varphi$ and $\psi$ such that the lifespan $T_{\varepsilon}$ of $u_{\varepsilon}$ satisfies the following inquality for $0<\varepsilon<\varepsilon_{0}$ :

$$
\begin{array}{ll}
T_{\varepsilon} \geq \exp \left(C \varepsilon^{-(p-1)}\right) & \text { for } p=p_{0}(2), \\
\varepsilon^{p-1} T_{\varepsilon}^{-q(2, p) / p} \log T_{\varepsilon} \geq C & \text { for } 2 \leq p<p_{0}(2), p \neq 3, \\
\varepsilon^{2} T_{\varepsilon}^{1 / 3}\left(\log T_{\varepsilon}\right)^{2} \geq C & \text { for } p=3 .
\end{array}
$$

THEOREM 2. Let $u_{\varepsilon}$ be a $C^{2}$-solution to (1.1) with initial data $f(x)=0$ and $g(x)=\varepsilon \psi(x)$, where $\varepsilon>0, \psi(x) \geq 0, \quad \equiv 0$ and $\phi \in C^{2}\left(\boldsymbol{R}^{2}\right)$. Then there exists a positive constant $C$ depending only on $p, A$ and $\psi$ such that the lifespan $T_{\varepsilon}$ of $u_{\varepsilon}$ has an upper bound:

$$
T_{\varepsilon} \leq C \varepsilon^{p(p-1) / q(2, p)} \quad \text { for } 1<p<p_{0}(2) .
$$

We give here some remarks. Firstly, H. Lindblad [9] has proved for $p=2$ that if $\int_{R^{2}} \psi(x) d x=0$ then $\lim _{\varepsilon \rightarrow 0} \varepsilon T_{\varepsilon}$ exists and if $\int_{R^{2}} \psi(x) d x \neq 0$ then $\lim _{\varepsilon \rightarrow 0} a(\varepsilon)^{-1} T_{\varepsilon}$ exists, where $a(\varepsilon)$ is defined by $a(\varepsilon)^{2} \varepsilon^{2} \log (a(\varepsilon)+1)=0$. His results are much sharper than ours. Secondly, making use of the method proving Theorem 1 or the one in K. Kubota [8], we can prove simply the existence of global solutions in R. T. Glassey [3] (see Appendix). Thirdly, we do not know the results of lower bounds of $T_{\varepsilon}$ for $1<p<2$. Finally the assumption of Theorem 2 does not require that the support of initial data is compact.

When the supports of initial data is non compact and $p>p_{0}(3), F$. Asakura [2] has proved in three space dimensions the following results. Let $D^{\alpha} f(x), D^{\beta} y(x)=O\left(|x|^{-1-\kappa}\right)$ as $|x| \rightarrow \infty \quad(|\alpha| \leq 3,|\beta| \leq 2)$. Then the global solution to (1.1) exists for small initial data provided $x>2 /(p-1)$. Moreover, he also proved that the lifespan of a solution to (1.1) is finite if $0<x<2 /(p-1)$ and initial data satisfy

$$
f(x)=0 \text { and } g(x) \geq \varepsilon(1+|x|)^{-1-\kappa} .
$$

The next aim of this paper is to show that, in two space dimensions, the lifespan is finite under the same assumption above. For global existence of solutions, see K. Kubota [8]

THEOREM 3. Let $u_{\varepsilon}$ be a $C^{2}$-solution to (1.1) with intial data satisfying (1.3). Then there exists a positive constant $C$ depending only on $A, p$ 
and $x$ such that the lifespan $T_{\varepsilon}$ of $u_{\varepsilon}$ has an upper bound:

$$
T_{\varepsilon} \leq C \varepsilon^{\left(\kappa-\frac{2}{p-1}\right)^{-1}} \text { for } p>1 \text { and } 0<x<\frac{2}{p-1} \text {. }
$$

We state here the relations between the upper bounds of $T_{\varepsilon}$ in Theorem 2 and Theorem 3 . Let $1<p<p_{0}(2)$, then we have

$$
\frac{1}{2}+\frac{1}{p}<\frac{2}{p-1}
$$

because

$$
\frac{q(2, p)}{p(p-1)}=\frac{1}{2}+\frac{1}{p}-\frac{2}{p-1}<0
$$

Therefore the upper bound in Theorem 2 (3) is better than one in Theorem $3(2)$ provided $1 / 2+1 p \leq x<2 /(p-1)(0<x<1 / 2+1 / p)$, respectively.

In $\S 2$, we define the norm to be used and formulate an a priori estimate which plays an important role in the proof of the existence theorem. Making use of a priori estimate, we prove Theorem 1, employing the iteration method in F. John [6]. In $\S 3$, we prove a priori estimate mentioned above. Theorem 2 will be proved in $\S 4$ by making use of the methods in F. John [6] and R. Agemi [1]. Theorem 3 will be also proved in $\S 5$ by the same method as in $\S 4$. In Appendix, we give a simple proof of the global existence theorem for $p>p_{0}(2)$.

After this work was completed, we were informed of a recent work of K. Tsutaya [11] closely related to our Theorem 3. He also proved the global existence mentioned before Theorem 3 by using different way from K. Kubota [8].

\section{$\S 2$. Proof of Theorem 1}

Let initial data $f(x)=\varepsilon \varphi(x)$ and $g(x)=\varepsilon \psi(x)$ be supported in $|x|<k$. Then we find from [7], Appendix (also see [1]) that a $C^{2}$-solution $u$ to (1. 1) with $p \geq 1$ is unique and

$$
u(x, t)=0 \quad \text { for } \quad|x|>t+k .
$$

Considering this fact, we difine the norm for a continuous function $u(x, t)$ in $Q_{T}=\boldsymbol{R}^{2} \times[0, T)$ satisfying (2.1) :

$$
\|u\|=\sup _{(x, t) \in Q_{T}} N(|x|, t)|u(x, t)|
$$

where 


$$
N(r, t)=k^{-(p+2) / 2 p}(t+r+2 k)^{1 / 2}(t-r+2 k)^{1 / p}, 2 \leq p \leq p_{0}(2) .
$$

As is well known, a solution to (1.1) has to satisfy the integral equation of the from

$$
u(x, t)=u_{0}(x, t)+A L\left(|u|^{p}\right)(x, t),
$$

where

$$
L\left(|u|^{p}\right)(x, t)=\frac{1}{2 \pi} \int_{0}^{t} d \tau \int_{0}^{t-\tau} \frac{\rho d \rho}{\sqrt{(t-\tau)^{2}-\rho^{2}}} \int_{|\omega|=1}|u|^{p}(x+\rho \omega, \tau) d S_{\omega}
$$

and $u_{0}$ is a solution to a linear wave equation

$$
\begin{aligned}
& \partial_{t}^{2} u_{0}(x, t)-\Delta u_{0}(x, t)=0, \\
& u_{0}(x, 0)=\varepsilon \varphi(x), \partial_{t} u_{0}(x, 0)=\varepsilon \phi(x) .
\end{aligned}
$$

By definition of the operator $L$, we see that if $u$ satisfies (2.1), then $L\left(|u|^{p}\right)$ has the same property of $u$.

Now we can formulate an a priori estimate which is a core in the proof of Theorem 1 .

Lemma 2.1. There exists a positive constant $C$ depending only on $p$ such that, for any continuous function $u(x, t)$ satisfying (2. 1),

$$
\left\|L\left(|u|^{p}\right)\right\| \leq C k^{2} M(k, p, T)\|u\|^{p} \quad \text { for } \quad 2 \leq p \leq p_{0}(2),
$$

where

$$
M(k, p, T)=\left\{\begin{array}{l}
\left(\frac{T+2 k}{k}\right)^{-q(2, p) / p} \log \frac{T+2 k}{k} \text { for } p \neq 3, \\
\left(\frac{T+2 k}{k}\right)^{1 / 3}\left(\log \frac{T+2 k}{k}\right)^{2} \text { for } p=3 .
\end{array}\right.
$$

Let $X$ denotes the Banach space of functions $u(x, t)$ for which the $D^{\alpha} u$ are continuous in $Q_{T}$ for $|\alpha| \leq 2$ and satisfy (2.1) and $\left\|D^{\alpha} u\right\|<\infty$. Here $D^{\alpha}$ stands for the space differentiation $D_{x_{1}}^{\alpha_{1}} D_{x_{2}}^{\alpha_{2}}$.

We shall look for a solution $u$ to (1.1) in $X$, employing the classical iteration method:

$$
u_{n+1}=u_{0}+A L\left(\left|u_{n}\right|^{p}\right), \quad n \in N .
$$

It follows from Lemma 1 in [3], p. 236 that a solution $u_{0}$ to (2.6) satisfies

$$
\left|D^{\alpha} u_{0}(x, t)\right| \leq C(\varphi, \phi) \varepsilon(t+r+2 k)^{-1 / 2}(t-r+2 k)^{-1 / 2}
$$

for $|\alpha| \leq 2$ and $t \geq 0$, where a positive constant $C(\varphi, \psi)$ depends on $D^{\alpha} \psi$ and $D^{\beta} \varphi$ for $|\beta| \leq 3$. Hence we find from (2.2), (2.3) and (2.10) that 
(2.11)

$$
\left\|D^{\alpha} u_{0}\right\| \leq C(\varphi, \psi) k^{-1} \varepsilon
$$

which implies $u_{0} \in X$. Here we have used the fact that $p \geq 2$ and $t-r$ $+2 k>k$.

Assume the a priori estimate (2.7). Then F. John [6] proved that the sequence $\left\{u_{n}\right\}$ defined by (2.9) converges in $X$ if $u_{0}$ satisfies the inequality (56e) in [6], p. 24. Applying the inequality to our case, we know that $\left\{u_{n}\right\}$ converges in $X$ if

$$
A C k^{2} M(k, p, T)\left\|u_{0}\right\|^{p-1} \leq \frac{1}{p 2^{p}},\left\|u_{0}\right\|<\frac{1}{2}
$$

for $2 \leq p \leq p_{0}(2)$. Therefore we find from (2.8) (2.11) that (2.12) holds if

$$
\begin{aligned}
& A C k^{2}\left(\frac{T+2 k}{k}\right)^{-q(2, p) / p} \log \frac{T+2 k}{k} \varepsilon^{p-1}\left(k^{-1} C(\varphi, \psi)\right)^{p-1} \leq \frac{1}{p 2^{p}} \text { for } p \neq 3, \\
& A C k^{2}\left(\frac{T+2 k}{k}\right)^{1 / 3}\left(\log \frac{T+2 k}{k}\right)^{2} \varepsilon^{2}\left(k^{-1} C(\varphi, \psi)\right)^{2} \leq \frac{1}{24} \text { for } p=3
\end{aligned}
$$

and

$$
C(\varphi, \psi) k^{-1} \varepsilon<\frac{1}{2} .
$$

Thus Theorem 1 is proved by taking $\varepsilon_{0}$ small.

\section{$\S 3$. Proof of the a priori estimate}

Let $u$ be a continuous function in $Q_{T}=\boldsymbol{R}^{2} \times[0, T)$ satisfying (2.1). Then $L\left(|u|^{p}\right)$ satisfies (2.1) and hence we can assume hereafter that

$$
r<t+k, \quad r=|x|
$$

It follows from (2.2) and (2.5) that

$$
\begin{aligned}
& \left|L\left(|u|^{p}\right)(x, t)\right| \\
& \leq \frac{\|u\|^{p}}{2 \pi} \int_{0}^{t} d \tau \int_{0}^{t-\tau} \frac{\rho d \rho}{\sqrt{(t-\tau)^{2}-\rho^{2}}} \int_{|\omega|=1} N(|x+\rho \omega|, \tau)^{-p} d S_{\omega} .
\end{aligned}
$$

The integral over the unit circle in (3.2) is equal to

$$
\begin{aligned}
& 2 \int_{0}^{\pi} N\left(\left(|x|^{2}+\rho^{2}+2 \rho|x| \cos \theta\right)^{1 / 2}, \tau\right)^{-p} d \theta \\
& =2 \int_{-1}^{1}\left(1-\eta^{2}\right)^{-1 / 2} N\left(\left(r^{2}+\rho^{2}+2 \rho r \eta\right)^{1 / 2}, \tau\right)^{-p} d \eta .
\end{aligned}
$$

Changing the variable $\eta$ for

$$
\lambda=\left(r^{2}+\rho^{2}+2 \rho r \eta\right)^{1 / 2},
$$


we find that the integral becomes

$$
4 \int_{|\rho-r|}^{\rho+r} \lambda N(\lambda, \tau)^{-p} h(\lambda, \rho ; r)^{-1 / 2},
$$

where

$$
h(\lambda, \rho ; r)=\left(\rho^{2}-(\lambda-r)^{2}\right)\left((\lambda+r)^{2}-\rho^{2}\right) .
$$

Therefore we get from (3.2)

$$
\begin{aligned}
& \left|L\left(|u|^{p}\right)(x, t)\right| \\
& \leq \frac{2\|u\|^{p}}{\pi} \int_{0}^{t} d \tau \int_{0}^{t-\tau} \frac{\rho d \rho}{\sqrt{(t-\tau)^{2}-\rho^{2}}} \int_{|\rho-r|}^{\rho+r} \frac{\lambda N(\lambda, \tau)^{-p}}{\sqrt{h(\lambda, \rho ; r)}} d \lambda .
\end{aligned}
$$

In what follows, we can assume that

(3.5) $\lambda<\tau+k$,

because of (2.1).

Inverting the order of the $(\rho, \lambda)$-integral, we find that the integral in (3. 4) is equal to

$$
\begin{aligned}
& \int_{0}^{t} d \tau \int_{|r-t+\tau|}^{r+t-\tau} \lambda N(\lambda, \tau)^{-p} d \lambda \int_{|\lambda-r|}^{t-\tau} \frac{\rho d \rho}{\sqrt{\left((t-\tau)^{2}-\rho^{2}\right) h(\lambda, \rho ; r)}} \\
& +\int_{0}^{t-r} d \tau \int_{0}^{t-\tau-r} \lambda N(\lambda, \tau)^{-p} d \lambda \int_{|\lambda-r|}^{\lambda+r} \frac{\rho d \rho}{\sqrt{\left((t-\tau)^{2}-\rho^{2}\right) h(\lambda, \rho ; r)}} \\
& =I_{1}(r, t)+I_{2}(r, t),
\end{aligned}
$$

where the last equality gives the difinitions of $I_{1}$ and $I_{2}$, and we regard $I_{2}$ as zero for $t<r$.

It follows from (2.2), (3.4) and (3.6) that the a priori estimate (2.7) in Lemma 2.1 is valid provided

$$
I_{j}(r, t) \leq C(p) k^{2} M(k, p, T) N(r, t)^{-1} \quad \text { for } \quad j=1.2,
$$

where $M$ is defined in (2.8) and we denote here and hereafter by $C(p)$ various positive constants depending only on $p$.

Firstly, we investigate the integral $I_{1}$. In the interval of the $\rho$-integral, we have

$$
\begin{aligned}
& \lambda+r-\rho \geq \lambda+r-t+\tau, \\
& \lambda+r+\rho \geq \lambda+r+|\lambda-r| \geq 2 \lambda \quad \text { or } \quad 2 r .
\end{aligned}
$$

Since 


$$
\int_{a}^{b} \frac{\rho d \rho}{\sqrt{\rho^{2}-a^{2}} \sqrt{b^{2}-\rho^{2}}}=\frac{1}{2} B\left(\frac{1}{2}, \frac{1}{2}\right)=\frac{\pi}{2} \quad \text { for } 0 \leq a<b,
$$

it follows from (3.3), (3.6), (3.8) and (3.9) with $a=|\lambda-r|, b=t-\tau$ that

$$
\int_{|\lambda-r|}^{t-\tau} \frac{\rho d \rho}{\sqrt{\left((t-\tau)^{2}-\rho^{2}\right) h(\lambda, \rho ; r)}} \leq \frac{\pi}{2 \sqrt{2}(\sqrt{r} \text { or } \sqrt{\lambda}) \sqrt{\tau+\lambda-t+r}}
$$

Thus we get

$$
I_{1}(r, t) \leq \frac{\pi}{2 \sqrt{2}} \int_{0}^{t} d \tau \int_{|r-t+\tau|}^{r+t-\tau} \frac{\lambda N(\lambda, \tau)^{-p}}{(\sqrt{r} \text { or } \sqrt{\lambda}) \sqrt{\tau+\lambda-t+r}} d \lambda
$$

Introducing new variables of integration

$$
\alpha=\tau+\lambda, \quad \beta=\tau-\lambda
$$

and extending the domain of $(\alpha, \beta)$-integration, we find from (3.5) and (3. 10) that

$$
I_{1}(r, t) \leq \frac{\pi}{4 \sqrt{2}} \int_{t-r}^{t+r} d \alpha \int_{-k}^{t-k} \frac{\lambda N(\lambda, \tau)^{-p}}{(\sqrt{r} \text { or } \sqrt{\lambda}) \sqrt{\alpha-(t-r)})} d \beta .
$$

We shall prove (3.7) for $I_{1}$ in the following two cases.

CASE 1: $\quad 4 r>t+r+2 k$.

Since $2 \lambda=\alpha-\beta<\alpha+k$ in the domain of integration and, by definition,

$$
N(\lambda, \tau)=k^{-(p+2) / 2 p}(\alpha+2 k)^{1 / 2}(\beta+2 k)^{1 / p},
$$

we get from (3.11)

$$
I_{1}(r, t) \leq \frac{\pi k^{(p+2) / 2}}{8 \sqrt{2 r}} \int_{t-r}^{t+r} \frac{(\alpha+2 k)^{(2-p) / 2}}{\sqrt{\alpha-(t-r)}} d \alpha \int_{-k}^{t-r}(\beta+2 k)^{-1} d \beta .
$$

Note that $\alpha-(t-r) \leq \alpha+k$, because of (3.1). Then the integration by parts yields

$$
\begin{aligned}
& \int_{t-r}^{t+r} \frac{(\alpha+2 k)^{(2-p) / 2}}{\sqrt{\alpha-(t-r)}} d \alpha \\
& \leq 2 \sqrt{2 r}(t+r+2 k)^{(2-p) / 2}+(p-2) \int_{t-r}^{t+r}(\alpha+2 k)^{(1-p) / 2} d \alpha .
\end{aligned}
$$

Let $3<p \leq p_{0}(2)$. Then it follows from (3.14) that the $\alpha$-integral in (3. 13) is dominated by

$$
2 \sqrt{2}(t+r+2 k)^{(3-p) / 2}+\frac{2(p-2)}{p-3}(t-r+2 k)^{(3-p) / 2}
$$




$$
\leq 2\left(\sqrt{2}+\frac{p-2}{p-3}\right)(t-r+2 k)^{(3-p) / 2} .
$$

Therefore, it follows from this, (1.2), (2.3) and (3.13) that

$$
\begin{aligned}
I_{1}(r, t) & \leq C(p) k^{(p+2) / 2}(t+r+2 k)^{-1 / 2}(t-r+2 k)^{(3-p) / 2} \log \frac{t-r+2 k}{k} \\
& =C(p) N(r, t)^{-1} k^{(p+2)(p-1) / 2 p}(t-r+2 k)^{-q(2, p) / p} \log \frac{t-r+2 k}{k} \\
& \leq C(p) k^{2} N(r, t)^{-1}\left(\frac{T+2 k}{k}\right)^{-q(2, p) / p} \log \frac{T+2 k}{k},
\end{aligned}
$$

which is (3.7) for $I_{1}$.

Let $2 \leq p<3$. Then it follows from (3.14) that the $\alpha$-integral in (3.13) is dominated by

$$
2\left(\sqrt{2}+\frac{p-2}{3-p}\right)(t+r+2 k)^{(3-p) / 2} \leq 2\left(\sqrt{2}+\frac{p-2}{3-p}\right)(2 t+3 k)^{(3-p) / 2} .
$$

Therefore, we get

$$
\begin{aligned}
I_{1}(r, t) & \leq C(p) k^{(p+2) / 2}(t+r+2 k)^{-1 / 2}(T+2 k)^{(3-p) / 2} \log \frac{T+2 k}{k} \\
& \leq C(p) k^{2} N(r, t)^{-1}\left(\frac{T+2 k}{k}\right)^{-q(2, p) / p} \log \frac{T+2 k}{k} .
\end{aligned}
$$

Let $p=3$. Then, in the same way as above, the $\alpha$-integral in (3.13) is dominated by

$$
2 \sqrt{2 r}(t+r+2 k)^{-1 / 2}+\log \frac{t+r+2 k}{t-r+2 k} \leq 2 \sqrt{2}+\log \frac{2 t+3 k}{k} .
$$

Therefore, we get

$$
\begin{aligned}
I_{1}(r, t) & \leq C(3) k^{5 / 2}(t+r+2 k)^{-1 / 2}\left(\log \frac{T+2 k}{k}\right)^{2} \\
& \leq C(3) k^{2} N(r, t)^{-1}\left(\frac{T+2 k}{k}\right)^{1 / 3}\left(\log \frac{T+2 k}{k}\right)^{2} .
\end{aligned}
$$

Thus we have proved (3.7) for $I_{1}$ in Case 1.

CASE 2: $\quad 4 r<t+r+2 k$, i.e., $t+r+2 k<2(t-r+2 k)$.

In this case, we choose $\sqrt{\lambda}$ in a denominator of the integrand in (3.

11). The we have

$$
I_{1}(r, t) \leq \frac{\pi}{8} k^{(p+2) / 2} \int_{t-r}^{t+r} \frac{(\alpha+2 k)^{(1-p) / 2}}{\sqrt{\alpha-(t-r)}} d \alpha \int_{-k}^{t-r}(\beta+2 k)^{-1} d \beta
$$


The $\alpha$-integral in (3.16) is dominated, in the same way as in Case 1, by

$$
2 \sqrt{2 r}(t+r+2 k)^{(1-p) / 2}+(p-1) \int_{t-r}^{t+r}(\alpha+2 k)^{-p / 2} d \alpha .
$$

Let $2<p \leq p_{0}(2)$. Since $(t+r+2 k)$ is equivalent to $(t-r+2 k)$ in Case 2 , we find from (3.17) that the $\alpha$-integral in (3.16) is dominated by

$$
C(p)(t+r+2 k)^{-1 / 2}(t-r+2 k)^{(3-p) / 2} .
$$

Therefore we have

$$
\begin{aligned}
I_{1}(r, t) & \leq C(p) k^{(2+p) / 2}(t+r+2 k)^{-1 / 2}(t-r+2 k)^{(3-p) / 2} \log \frac{t-r+2 k}{k} \\
& \leq C(p) k^{2} N(r, t)^{-1}\left(\frac{T+2 k}{k}\right)^{-q(2, p) / p} \log \frac{T+2 k}{k} .
\end{aligned}
$$

Let $p=2$. Then the $\alpha$-integral in (3.16) is dominated by

$$
2 \sqrt{2 r}(t+r+2 k)^{-1 / 2}+\log \frac{t+r+2 k}{t-r+2 k} \leq 2 \sqrt{2}+\log 2
$$

Therefore, we have

$$
\begin{aligned}
I_{1}(r, t) & \leq C(2) k^{2} \log \frac{T+2 k}{k} \\
& \leq C(2) k^{2} N(r, t)^{-1}\left(\frac{T+2 k}{k}\right) \log \frac{T+2 k}{k} .
\end{aligned}
$$

Thus the proof of (3.7) for $I_{1}$ is finished.

Next we investigate the intergral $I_{2}$ defined for $t>r$. In the domain of the integraion, we have

$$
\begin{aligned}
& t-\tau-\rho \geq t-\tau-(\lambda+r)=t-r-\alpha \\
& t-\tau+\rho \geq t-\tau+|\lambda-r| \geq 2 r \text { or } t-r-\beta .
\end{aligned}
$$

It follows from this, (3.3) and (3.9) with $a=|\lambda-r|, b=\lambda+r$ that

$$
\int_{|\lambda-r|}^{\lambda+r} \frac{\rho d \rho}{\sqrt{\left((t-\tau)^{2}-\rho^{2}\right) h(\lambda, \rho ; r)}} \leq \frac{\pi}{2(\sqrt{2 r} \text { or } \sqrt{t-r-\beta}) \sqrt{t-r-\alpha})} .
$$

Hence we have from this and (3.6)

$$
I_{2}(r, t) \leq \frac{\pi}{2} \int_{0}^{t-r} d \tau \int_{0}^{t-\tau-r} \frac{\lambda N(\lambda, \tau)^{-p} d \lambda}{(\sqrt{2 r} \text { or } \sqrt{t-r-\beta}) \sqrt{t-r-\alpha})} .
$$

Extending the domain of $(\alpha, \beta)$-intergral, we find from (3.5) and (3.18) that 


$$
I_{2}(r, t) \leq \frac{\pi}{4} \int_{0}^{t-r} d \alpha \int_{-k}^{t-r} \frac{\lambda N(\lambda, \tau)^{-p} d \beta}{(\sqrt{2 r} \text { or } \sqrt{t-r-\beta}) \sqrt{t-r-\alpha}} .
$$

Since $2 \lambda=\alpha-\beta<\alpha+k$, we get from this and (3.12)

$$
I_{2}(r, t) \leq \frac{\pi k^{(2+p) / 2}}{8 \sqrt{2 r}} \int_{0}^{t-r} \frac{(\alpha+2 k)^{(2-p) / 2}}{\sqrt{t-r-\alpha}} d \alpha \int_{-k}^{t-r}(\beta+2 k)^{-1} d \beta
$$

or

$$
I_{2}(r, t) \leq \frac{\pi k^{(2+p) / 2}}{8} \int_{0}^{t-r} \frac{(\alpha+2 k)^{(2-p) / 2}}{\sqrt{t-r-\alpha}} d \alpha \int_{-k}^{t-r} \frac{(\beta+2 k)^{-1}}{\sqrt{t-r-\beta}} d \beta .
$$

We first show that, for $2 \leq p \leq p_{0}(2)$,

$$
\int_{0}^{t-r} \frac{(\alpha+2 k)^{(2-p) / 2}}{\sqrt{t-r-\alpha}} d \alpha \leq C(p)(t-r+2 k)^{(3-p) / 2} .
$$

In fact, let $0<t-r<k$, i.e., $3 k>\mathrm{t}-r+2 k$. Then, by integration by parts, the $\alpha$-integral is dominated by

$$
2 \sqrt{t-r}(2 k)^{(2-p) / 2} \leq 2\left(\frac{2}{3}\right)^{(2-p) / 2}(t-r+2 k)^{(3-p) / 2},
$$

where we have used the fact that $p \geq 2$. Let $t-r>k$ which implies $t-r>$ $(t-r+2 k) / 4$. Then, breaking the integral up into two pieces, we get

$$
\begin{aligned}
\int_{0}^{(t-r) / 2} \frac{(\alpha+2 k)^{(2-p) / 2}}{\sqrt{t-r-\alpha}} d \alpha & \leq \sqrt{2}(t-r)^{-1 / 2} \int_{0}^{(t-r) / 2}(\alpha+2 k)^{(2-p) / 2} d \alpha \\
& \leq \frac{4 \sqrt{2}}{4-p}(t-r+2 k)^{(3-p) / 2},
\end{aligned}
$$

because $p_{0}(2)<4$. On the complement,

$$
\begin{aligned}
\int_{(t-r) / 2}^{(t-r)} \frac{(\alpha+2 k)^{(2-p) / 2}}{\sqrt{t-r-\alpha}} d \alpha & \leq\left(\frac{t-r}{2}+2 k\right)^{(2-p) / 2} \int_{(t-r) / 2}^{t-r} \frac{d \alpha}{\sqrt{t-r-\alpha}} \\
& \leq \sqrt{2} 2^{(p-2) / 2}(t-r+2 k)^{(3-p) / 2} .
\end{aligned}
$$

Next we show in a similar way to the above that

$$
\int_{-k}^{(t-r)} \frac{(\beta+2 k)^{-1}}{\sqrt{t-r-\beta}} d \beta \leq C(t-r+2 k)^{-1 / 2} \log \frac{t-r+2 k}{k} .
$$

In fact, let $0<t-r<k$. Then, by integration by parts, the $\beta$-integral is dominated by

$$
2 \sqrt{t-r+k} k^{-1} \leq 6(t-r+2 k)^{-1 / 2} .
$$

Let $k<t-r$. Then, breaking the integral up into two pieces, we get 


$$
\begin{aligned}
\int_{-k}^{(t-r) / 2} \frac{(\beta+2 k)^{-1}}{\sqrt{t-r-\beta}} d \beta & \leq \sqrt{2}(t-r)^{-1 / 2} \int_{-k}^{(t-r) / 2}(\beta+2 k)^{-1} d \beta \\
& \leq 2 \sqrt{2}(t-r+2 k)^{-1 / 2} \log \frac{t-r+4 k}{2 k}
\end{aligned}
$$

On the complement,

$$
\begin{aligned}
\int_{(t-r) / 2}^{t-r} \frac{(\beta+2 k)^{-1}}{\sqrt{t-r-\beta}} d \beta & \leq\left(\frac{t-r}{2}+2 k\right)^{-1} \int_{(t-r) / 2}^{t-r} \frac{1}{\sqrt{t-r-\beta}} d \beta \\
& \leq 2 \sqrt{2}(t-r+2 k)^{-1 / 2} .
\end{aligned}
$$

When $4 r>t+r+2 k$, it follows from (3.19) and (3.21) that

$$
I_{2}(r, t) \leq C(p) k^{(2+p) / 2}(t+r+2 k)^{-1 / 2}(t-r+2 k)^{(3-p) / 2} \log \frac{T+2 k}{k} .
$$

When $4 r<t+r+2 k$, i.e., $t+r+2 k<2(t-r+2 k)$, we obtain (3.23) from (3.20), (3.21) and (3.22). Thus the estimate (3.7) for $I_{2}$ follows from (3. 23), by the same way as in (3.15). Therefore, the proof of Lemma 2.1 is completed.

\section{$\S 4$ Proof of Theorem 2}

Let $u(x, t)$ be a $C^{2}$-solution to $(1.1)$ in $\boldsymbol{R}^{2} \times[0, T)$ with the initial data defined in the statement of Theorem 2. In this section we shall show that if $T$ exceeds a certain constant we get a contradiction. This implis that the lifespan of $u$ has an upper bound which is expressed by such a constant.

By the assumption of Theorem 2 on $\psi$, there exists a $x_{0} \in \boldsymbol{R}^{2}$ such that (4. 1) $\quad \psi\left(x_{0}\right)>0$.

Throughout this section we use the following notation. Let $\bar{v}$ be the spherical mean of $v \in C^{0}\left(\boldsymbol{R}^{2} \times[0, \infty)\right)$ at a point $x_{0}$ with radius $r$;

$$
\bar{v}(r, t)=\frac{1}{2 \pi} \int_{|\omega|=1} v\left(x_{0}+r \omega, t\right) d S_{\omega} .
$$

Then (4.1) yields

$$
\bar{\psi}(0)>0 \text {. }
$$

Hence one can find $\delta>0$ so small that

(4. 4) $\quad \bar{\psi}(2 \delta)>0$.

As in section 2, we know that $u$ satisfies the integral equation (2. 4). Here we employ the following lemmas. 
LEMMA 4.1. Let $u_{0}$ be a solution to (2.6) with $\varphi(x)=0$. Then

$$
\overline{u_{0}}(r, t) \geq \frac{\varepsilon}{2 \pi \sqrt{r}} \int_{|t+r|}^{t+r} \sqrt{\lambda} \bar{\psi}(\lambda) d \lambda \text { for } 0<t \leq 2 r .
$$

LEMMA 4.2. Let $u$ be the solution to (1.1) with $f(x)=0, g(x) \geq 0$ and $p>1$.

Then

$$
\bar{u}(r, t) \geq \frac{A}{2 \pi \sqrt{r}} \iint_{T_{r, t}} \sqrt{\lambda}|\bar{u}|^{p}(\lambda, \tau) d \lambda d \tau
$$

for $0<t-r \leq r$, where

$$
T_{r, t}=\left\{(\lambda, \tau) \in \boldsymbol{R}^{2} ; t-r \leq \tau+\lambda \leq t+r, \tau-\lambda \leq t-r, \tau \geq 0\right\} .
$$

These lemmas are due to R. Agemi [1].

Lemma 4.2 follows from the proof of (2.11) in [1]. Applying its argument to $u_{0}$, we readily get Lemma 4 . 1. For the sake of completeness, we shall review proofs of these lemmas.

PROOF OF LEMMA 4.2: It follows from (2.4) that

$$
\bar{u}(r, t)=\overline{u_{0}}(r, t)+\overline{A L\left(|u|^{p}\right)}(r, t) .
$$

Since the assumption that $f(x)=0, g(x) \geq 0$ yields $\overline{u_{0}} \geq 0$, we have

$$
\bar{u}(r, t) \geq \overline{A L\left(|u|^{p}\right)}(r, t) .
$$

We now employ the following fundamental identity for iterated spherical means by F. John [5], p. 81 ;

$$
\begin{gathered}
\frac{1}{(2 \pi)^{2}} \int_{|\eta|=1} \int_{|\omega|=1} v(r \eta+\rho \omega) d S_{\omega} d S_{\eta} \\
\quad=\frac{2}{\pi} \int_{|\rho-r|}^{\rho+r} \frac{\lambda \bar{v}(\lambda)}{\sqrt{h(\lambda, \rho ; r)}} d \lambda,
\end{gathered}
$$

where $h(\lambda, \rho ; r)$ is defined by (3.3). Applying (4.10) with $v=|u|^{p}$ to the right hand side of (4.9), we get

$$
\bar{u}(r, t) \geq \frac{2 A}{\pi} \int_{0}^{t} d \tau \int_{0}^{t-\tau} \frac{\rho d \rho}{\sqrt{(t-\tau)^{2}-\rho^{2}}} \int_{|\rho-r|}^{\rho+r} \frac{\lambda|\bar{u}|^{p}(\lambda, \tau)}{\sqrt{h(\lambda, \rho ; r)}} d \lambda .
$$

Here we have used the Jensen's inequality

$$
\overline{|u|^{p}} \geq|\bar{u}|^{p} \quad \text { for } \quad p>1 \text {. }
$$

Note that 


$$
t-\tau-r \leq r \text { for } 0<t-r \leq r \text { and } \tau \geq 0 .
$$

Then, inverting the order of $(\lambda, \rho)$-integral, we find that the right hand side of (4.11) equals to

$$
\begin{aligned}
& \frac{2 A}{\pi} \int_{0}^{t} d \tau \int_{|t-\tau-r|}^{t-\tau-r} \lambda|\bar{u}|(\lambda, \tau) d \lambda \int_{|\lambda-r|}^{t-\tau} \frac{\rho d \rho}{\sqrt{h(\lambda, \rho ; r)\left((t-\tau)^{2}-\rho^{2}\right)}} \\
& +\frac{2 A}{\pi} \int_{0}^{t-r} d \tau \int_{0}^{t-\tau-r} \lambda|\bar{u}|^{p}(\lambda, \tau) d \lambda \int_{|\lambda-r|}^{\lambda+r} \frac{\rho d \rho}{\sqrt{h(\lambda, \rho ; r)\left((t-\tau)^{2}-\rho^{2}\right)}}
\end{aligned}
$$

Hence it follows from this and (4.11) that

$$
\bar{u}(r, t)
$$

$$
\geq \frac{2 A}{\pi} \int_{0}^{t} d \tau \int_{|t-\tau-r|}^{t-\tau+r} \lambda|\bar{u}|^{p}(\lambda, \tau) d \lambda \int_{|\lambda-r|}^{t-r} \frac{\rho d \rho}{\sqrt{h(\lambda, \rho: r)\left(\left(t-\tau^{\prime 2}-\rho^{2}\right)\right.}} .
$$

In the domain of $(\rho, \lambda)$-integral of (4.12), we know that

$$
h(\lambda, \rho ; r) \leq 12 r \lambda\left((t-\tau)^{2}-(\lambda-r)^{2}\right) \text { for } 0<t-r \leq r .
$$

In fact, $\rho \leq t-\tau$ implies

$$
\rho^{2}-(\lambda-r)^{2} \leq(t-\tau)^{2}-(\lambda-r)^{2} .
$$

Since $\lambda \leq t+r, \rho \leq t-\tau$ and $t \leq 2 r$, we have

$$
\lambda+r+\rho \leq t+r+r+t-\tau \leq 2 t+2 r \leq 6 r .
$$

Moreover, $\rho \geq|\lambda-r|$ yields

$$
\lambda+r-\rho \leq \lambda+r-|\lambda-r| \leq \lambda+r+\lambda-r=2 \lambda .
$$

Thus we get (4.13). Using the fact that

$$
\int_{|\lambda-r|}^{t-r} \frac{\rho d \rho}{\sqrt{(t-r)^{2}-\rho^{2}}}=\sqrt{(t-\tau)^{2}-(\lambda-r)^{2}},
$$

we obtain (4.6) by (4.12) and (4.13).

PROOF OF LEMMA 4.1: As is well known, a solution to (2.6) with $\varphi(x)=0$ is expressed in the form

$$
u_{0}(x, t)=\frac{1}{2 \pi} \int_{0}^{t} \frac{\rho d \rho}{\sqrt{t^{2}-\rho^{2}}} \int_{|\omega|=1} \varepsilon \psi(x+\rho \omega) d S_{\omega} .
$$

Applying (4.10) with $v=\psi$ to the right hand side of (4.14), we get 
(4. 15) $\quad \overline{u_{0}}(r, t)=\frac{2 \varepsilon}{\pi} \int_{0}^{t} \frac{\rho d \rho}{\sqrt{t^{2}-\rho^{2}}} \int_{|\rho-r|}^{\rho+r} \frac{\lambda \bar{\psi}(\lambda)}{\sqrt{h(\lambda, \rho ; r)}} d \lambda$.

As in the proof of Lemma 4.2, inverting the order of $(\lambda, \rho)$-integral yields for $0<t-r \leq r$

(4. 16) $\quad \overline{u_{0}}(r, t)=\frac{2 \varepsilon}{\pi} \int_{|t-r|}^{t+r} \lambda \bar{\psi}(\lambda) d \lambda \int_{|\lambda-r|}^{t} \frac{\rho d \rho}{\sqrt{h(\lambda, \rho ; r)\left(t^{2}-\rho^{2}\right)}}$.

Replacing $t-\tau$ by $t$ in (4.13), we obtain (4.5) by same way as in the proof of Lemma 4.2.

Now, define the region

$$
S=\left\{(r, t) \in \boldsymbol{R}^{2} ; 3 \delta \leq t+r, \delta \leq t-r \leq 2 \delta\right\},
$$

where $\delta$ is the one in (4.4). Then it follows from (4.4), (4.5) and (4.17)

(4.18) $\overline{u_{0}}(r, t) \geq \frac{C^{\prime} \varepsilon}{\sqrt{r}}$ for $(r, t) \in S$ and $t \leq 2 r$,

where $C^{\prime}$ is a constant defined by

(4. 19) $\quad C^{\prime}=\frac{1}{2 \pi} \int_{2 \delta}^{3 \delta} \sqrt{\lambda} \bar{\phi}(\lambda) d \lambda$.

Since (2.4) yields

(4.20) $\bar{u}(r, t) \geq \overline{u_{0}}(r, t)$,

we have

(4.21) $\quad \bar{u}(r, t) \geq \frac{C^{\prime} \varepsilon}{\sqrt{r}}$ for $(r, t) \in S$ and $t \leq 2 r$.

Let $\Sigma$ denote the set

(4.22) $\Sigma=\left\{(r, t) \in \boldsymbol{R}^{2} ; 3 \delta \leq t-r \leq r\right\}$.

For $(r, t) \in \Sigma$ we introduce the sets

$$
\begin{aligned}
& S_{r, t}=\{(\lambda, \tau) ; t-r \leq \lambda, \tau+\lambda \leq t+r, \delta \leq \tau-\lambda \leq 2 \delta\}, \\
& R_{r, t}=\{(\lambda, \tau) ; t-r \leq \lambda, \tau+\lambda \leq t+r, 3 \delta \leq \tau-\lambda \leq t-r\},
\end{aligned}
$$

We note that for $(r, t) \in \Sigma$

(4.24) $S_{r, t}, R_{r, t} \subset T_{r, t}, S_{r, t} \subset S, R_{r, t} \subset \Sigma$.

Hence it follows from (4.6), (4.21) and (4.24) that 


$$
\bar{u}(r, t) \geq \frac{A}{2 \pi \sqrt{r}} \iint_{S_{r, t}} \sqrt{\lambda}\left(\frac{C^{\prime} \varepsilon}{\sqrt{\lambda}}\right)^{p} d \lambda d \tau \quad \text { for } \quad(r, t) \in \Sigma
$$

Changing the variables by

$$
\alpha=\tau+\lambda, \quad \beta=\tau-\lambda
$$

we have for $(r, t) \in \Sigma$

$$
\bar{u}(r, t) \geq \frac{A\left(C^{\prime}\right)^{p}}{4 \pi \sqrt{r}} \varepsilon^{p} \int_{\delta}^{2 \delta} d \beta \int_{2(t-r)+\beta}^{t+r}\left(\frac{\alpha-\beta}{2}\right)^{(1-p) / 2} d \alpha .
$$

Since $p>1$ and $\alpha-\beta \leq \alpha \leq t+r \leq 3 r$,

$$
\bar{u}(r, t) \geq \frac{A\left(C^{\prime}\right)^{p}}{4 \pi}\left(\frac{2}{3}\right)^{(p-1) / 2} \varepsilon^{p} r^{-p / 2} \int_{\delta}^{2 \delta}(3 r-t-\beta) d \beta .
$$

Here we find that for $(r, t) \in \Sigma$

$$
3 r-t-\beta \geq r-\beta \geq r-2 \delta \geq r / 3
$$

Hence

$$
\bar{u}(r, t) \geq \frac{\delta A\left(C^{\prime}\right)^{p}}{12 \pi}\left(\frac{2}{3}\right)^{(p-1) / 2} \varepsilon^{p} r^{(2-p) / 2}
$$

Therefore we obtain for $(r, t) \in \Sigma$

(4.26) $\quad \bar{u}(r, t) \geq\left\{\begin{array}{l}C_{0} \varepsilon^{p} r^{-1 / 2}(t-r-s)^{(3-p) / 2} \text { if } 1<p<3, \\ C_{0} \varepsilon^{p} r^{-(p-2) / 2} \text { if } 3 \leq p<p_{0}(2),\end{array}\right.$

where we set $s=3 \delta$ and

$$
C_{0}=\frac{\delta A\left(C^{\prime}\right)^{p}}{12 \pi}\left(\frac{2}{3}\right)^{(p-1) / 2}
$$

Now, assume that $\bar{u}$ has more general estimate

$$
\bar{u}(r, t) \geq C r^{-q}(t-r-s)^{a}(t-r)^{-b} \text { for } \quad(r, t) \in \Sigma
$$

with $C>0, q \geq 1 / 2, a \geq 0, b \geqq 0$. Then it follows from (4.6), (4.24), (4.25) and (4.28) that for $(r, t) \in \Sigma$

$$
\begin{aligned}
\bar{u}(r, t) & \geq \frac{A}{2 \pi \sqrt{r}} \iint_{R_{r, t}} \sqrt{\lambda}|\bar{u}(\lambda, \tau)|^{p} d \lambda d \tau \\
& \geq \frac{A C^{p}}{2 \pi \sqrt{r}} \iint_{R_{r, t}} \lambda^{1 / 2-p q}(\tau-\lambda-s)^{p a}(\tau-\lambda)^{-p b} d \lambda d \tau \\
& \geq \frac{A C^{p}}{4 \pi \sqrt{r}} \int_{s}^{t-r} d \beta \int_{2(t-r)+\beta}^{t+r}\left(\frac{\alpha-\beta}{2}\right)^{1 / 2-p q}(\beta-s)^{p a} \beta^{-p b} d \alpha .
\end{aligned}
$$


We divide the estimate for $\bar{u}$ into the following two cases.

CASE 1: $\quad t-r \geq r / 2$.

Since $p q>q>1 / 2$ and

$$
\alpha-\beta \leq \alpha \leq t+r \leq 3 r \leq 6(t-r)
$$

for $(r, t) \in \Sigma$ in this case, we have

$$
\bar{u}(r, t) \geq \frac{3^{1 / 2-p q} A C^{p}}{4 \pi \sqrt{r}(t-r)^{p b+p q-1 / 2}} \int_{s}^{t-r}(\beta-s)^{p a}(3 r-t-\beta) d \beta .
$$

By $3 r-t \geq r \geq t-r, \beta$-integral is replaced gy

$$
\int_{s}^{t-r}(\beta-s)^{p a}(t-r-\beta) d \beta=\frac{(t-r-s)^{p a+2}}{(p a+1)(p a+2)} .
$$

Hence

$$
\bar{u}(r, t) \geq \frac{3^{1 / 2-p q} A C^{p}(t-r-s)^{p a+2}}{4 \pi(p a+2)^{2} \sqrt{r}(t-r)^{p b+p q-1 / 2}} .
$$

CASE 2: $t-r \leq / 2$.

Since $t+r \geq 2 r \geq 4(t-r)$ and $2(t-r)+\beta \leq 3(t-r)$ for $(r, t) \in \Sigma$ in this case, we have

$$
\begin{aligned}
\bar{u}(r, t) & \geq \frac{A C^{p}}{4 \pi \sqrt{r}} \int_{s}^{t-r}(\beta-s)^{p a} \beta^{-p b} d \beta \int_{3(t-r)}^{4(t-r)}\left(\frac{\alpha}{2}\right)^{1 / 2-p q} d \alpha \\
& \geq \frac{2^{1 / 2-p q} A C^{p}}{4 \pi \sqrt{r}(t-r)^{p b+p q-3 / 2}} \int_{s}^{t-r}(\beta-s)^{p a} d \beta \\
& \geq \frac{2^{1 / 2-p q} A C^{p}(t-r-s)^{p a+1}}{4 \pi(p a+1) \sqrt{r}(t-r) p^{p b+p q-3 / 2}} \\
& \geq \frac{3^{1 / 2-p q} A C^{p}(t-r-s)^{p a+2}}{4 \pi(p a+2)^{2} \sqrt{r}(t-r)^{p b+p q-1 / 2}}
\end{aligned}
$$

Combining these two cases, we obtain

$$
\bar{u}(r, t) \geq C^{*} r^{-1 / 2}(t-r-s)^{a^{*}}(t-r)^{-b^{*}} \text { for }(r, t) \in \Sigma,
$$

where

$$
\begin{array}{ll}
a^{*}=p a+2, & b^{*}=p b+p q-\frac{1}{2}, \\
C^{*}=\frac{D_{q} C^{p}}{(p a+2)^{2}}, & D_{q}=\frac{3^{1 / 2-p q} A}{4 \pi}
\end{array}
$$

Using the values 
(4. 31)

$$
\begin{aligned}
& q=\frac{1}{2}, a=\frac{3-p}{2}, b=0, C=C_{0} \varepsilon^{p} \quad \text { if } \quad 1<p<3, \\
& q=\frac{p-2}{2}, a=b=0, C=C_{0} \varepsilon^{p} \quad \text { if } \quad 3 \leq p<p_{0}(2)
\end{aligned}
$$

corresponding to (4.26), we have (4.29) with

$$
\begin{aligned}
& a^{*}=\frac{p(3-p)}{2}+2, b^{*}=\frac{p-1}{2}, C^{*}=\frac{D_{1 / 2}\left(C_{0} \varepsilon^{p}\right)^{p}}{(p(3-p) / 2+2)^{2}} \quad \text { if } \quad 1<p<3, \\
& a^{*}=2, \quad b^{*}=\frac{p(p-2)-1}{2}, C^{*}=\frac{D_{(p-2) / 2}\left(C_{0} \varepsilon^{p}\right)^{p}}{4} \text { if } 3 \leq p \leq p_{0}(2)
\end{aligned}
$$

Define the sequences $\left\{a_{\ell}\right\},\left\{b_{\ell}\right\},\left\{C_{\ell}\right\}(\ell \in \boldsymbol{N})$ by

$$
\begin{aligned}
& a_{\ell+1}=p a_{\ell}+2, \quad b_{\ell+1}=p b_{\ell}+\frac{p-1}{2}, C_{\ell+1}=\frac{D_{1 / 2} C_{\ell}^{p}}{\left(p a_{\ell}+2\right)^{2}}, \\
& a_{1}=a^{*}, b_{1}=b^{*}, C_{1}=C^{*} \quad \text { as given in }(4.32) .
\end{aligned}
$$

Then (4.29) will hold with $q=1 / 2, a^{*}=a_{\ell}, b^{*}=b_{\ell}, C^{*}=C_{\ell}$ for $\ell \in \boldsymbol{N}$. Solving the above sequences we have

$$
\begin{aligned}
& a_{\ell}=\left(\frac{3-p}{2}+\frac{2}{p-1}\right) p^{\ell}-\frac{2}{p-1}, b_{\ell}=\frac{1}{2} p^{\ell}-\frac{1}{2} \quad \text { if } \quad 1<p<3, \\
& a_{\ell}=\frac{2}{p-1} p^{\ell}-\frac{2}{p-1}, \mathrm{~b}_{\ell}=\frac{p-2}{2} p^{\ell}-\frac{1}{2} \quad \text { if } \quad 3 \leq p<p_{0}(2) .
\end{aligned}
$$

If $1<p<3$, then

$$
\begin{aligned}
p a_{\ell}+2 & =\left(\frac{3-p}{2}+\frac{2}{p-1}\right) p^{\ell+1}-\frac{2}{p-1} . \\
& \leq \frac{2}{p-1}\left(p^{\ell+2}-1\right) \leq 2(\ell+2) p^{\ell+1} .
\end{aligned}
$$

If $3 \leq p<p_{0}(2)$, then

$$
p a_{\ell}+2=\frac{2}{p-1}\left(p^{\ell+1}-1\right) \leq 2(\ell+2) p^{\ell+1} .
$$

Hence it follows from (4.33) that

$$
C_{\ell+1} \geq \frac{D_{1 / 2} C_{\ell}^{p}}{4(\ell+2)^{2} p^{2 \ell+2}} \quad \text { for } \quad 1<p<p_{0}(2),
$$

which implies that

$$
C_{\ell} \geq \exp \left[p^{\ell}\left(\frac{1}{p} \log C_{1}-\sum_{j=1}^{\ell-1} \frac{2 \log (j+2)+2(j+1) \log p+\log 4 D_{1 / 2}^{-1}}{p^{j+1}}\right)\right]
$$

For sufficiently large $\ell$ we have 
(4. 35)

$$
C_{\ell} \geq \exp \left[p^{\ell}\left(p^{-1} \log C_{1}-S(p)\right)\right] \text { for } 1<p<p_{0}(2)
$$

where

(4. 36) $S(p)=\sum_{j=1}^{\infty} \frac{2 \log (j+2)+2(j+1) \log p+\log 4 D_{1 / 2}^{-1}}{p^{j+1}}$.

We note that $S(p)$ is finite because $p>1$ and each term is positive for sufficiently large $\ell$. Therefore it follows from (4.29), (4.33), (4.34) and (4. 35) that for $(r, t) \in \Sigma$

$$
\bar{u}(r, t) \geq \frac{\sqrt{t-r}}{\sqrt{r}(t-r-s)^{2 /(p-1)}} \exp \left[p^{\ell} J(r, t)\right]
$$

where

$$
\begin{aligned}
& J(r, t)=p^{-1} \log C_{1}-S(p)+ \\
& +\left\{\begin{array}{l}
\left(\frac{3-p}{2}+\frac{2}{p-1}\right) \log (t-r-s)-\frac{1}{2} \log (t-r) \quad \text { if } \quad 1<p<3, \\
\frac{2}{p-1} \log (t-r-s)-\frac{p-2}{2} \log (t-r) \text { if } 3 \leq p<p_{0}(2) .
\end{array}\right.
\end{aligned}
$$

If there exists a point $(r, t) \in \Sigma$ such that

$$
\text { (4. 39) } J(r, t)>0 \text {, }
$$

then we have $\bar{u}(r, t)=\infty$ letting $\ell \rightarrow \infty$, which implies that $u$ cannot be a $C^{2}$-solution to (1.1). This contradicts the assumption that $u$ is a $C^{2}$-solution to (1.1), so that we shall look for $(r, t) \in \Sigma$ which satisfies (4.39). In view of (4.38), if $t-r \geq 2 s$, (4.39) follows from

$$
\begin{gathered}
\left(\frac{2}{p-1}-\frac{p-2}{2}\right) \log (t-r)>-\frac{1}{p} \log C_{1}+S(p)+\frac{2 p}{p-1} \log 2 \\
\text { for } 1<p<p_{0}(2) .
\end{gathered}
$$

Hence the definition of $C_{1},(4.33)$, gives the following sufficient condition to $(4.39)$;

$$
\frac{q(2, p)}{1-p} \log (t-r)>\log C^{\prime \prime} \varepsilon^{-p} \quad \text { for } \quad 1<p<p_{0}(2),
$$

where $q(2, p)$ is defined by (1.2) and we set

$$
C^{\prime \prime}=\left(5^{2} D_{1}^{-1}\right)^{1 / p} C_{0}^{-1} e^{S(p)+2 p /(p-1)} .
$$

$C_{0}$ is defined by (4.27). Here we have used the following fact which follows from the definition of $D_{q},(4.30)$.

(4. 41) $D_{1 / 2} \geq D_{q}$ for $q \geq 1 / 2$. 
Recall that $q(2, p)<0$ for $1<p<p_{0}(2)$. Therefore, setting $t=2 r$, we find that (4. 39) follows from

$$
t>C \varepsilon^{p(p-1) / q(2, p)} \text { for } 1<p<p_{0}(2),
$$

where

$$
C=2\left(C^{\prime \prime}\right)^{(1-p) / q(2, p)}
$$

Thus we conclude that $T$ has to satisfy

$$
T \leq C \varepsilon^{p(p-1) / q(2, p)} \text {. }
$$

This completes the proof of Theorem 2 .

\section{$\S 5$. Proof of Theorem 3}

In this section we shall prove Theorem 3 by using the same argument as in the proof of Theorem 2.

Let $u(x, t)$ be a $C^{2}$-solution to $(1.1)$ in $\boldsymbol{R}^{2} \times[0, T)$ with initial data satisfying (1.3). In the definition of the spherical mean, (4.2), we set $x_{0}=$ 0 . Define the set

$$
\Sigma^{\prime}=\left\{(r, t) \in \boldsymbol{R}^{2} ; s<t-r \leq r\right\}
$$

for some fixed constant $s>0$. By virture of Lemma 4.1, (4.5), we have for $(r, t) \in \Sigma^{\prime}$

$$
\begin{aligned}
\overline{u_{0}}(r, t) & \geq \frac{\varepsilon}{2 \pi \sqrt{r}} \int_{t-r}^{t+r} \sqrt{\lambda} \bar{\psi}(\lambda) d \lambda \\
& \geq \frac{\varepsilon}{2 \pi \sqrt{r}} \int_{t-r}^{t+r} \sqrt{\lambda}(1+\lambda)^{-\kappa-1} d \lambda \\
& \geq \frac{1}{2 \pi}\left(\frac{s}{1+s}\right)^{\kappa-1} \frac{\varepsilon}{\sqrt{r}} \int_{t-r}^{t+r} \lambda^{-k-1 / 2} d \lambda
\end{aligned}
$$

If $0<x \leq 1 / 2$, then

$$
\begin{aligned}
\overline{u_{0}}(r, t) & \geq \frac{1}{2 \pi}\left(\frac{s}{1+s}\right)^{\kappa+1} \frac{\varepsilon}{\sqrt{r}(t+r)^{\kappa+1 / 2}} \int_{t-r}^{t+r} d \lambda \\
& \geq \frac{1}{3 \pi}\left(\frac{s}{1+s}\right)^{\kappa+1} \frac{\varepsilon}{r^{\kappa} .}
\end{aligned}
$$

If $x>1 / 2$, then

$$
\begin{aligned}
\overline{u_{0}}(r, t) & \geq \frac{1}{\pi(2 \varkappa-1)}\left(\frac{s}{1+s}\right)^{\kappa+1} \frac{\varepsilon}{\sqrt{r}(t-r)^{\kappa-1 / 2}}\left[1-\left(\frac{t-r}{t+r}\right)^{\kappa-1 / 2}\right] \\
& \geq \frac{1}{\pi(2 \varkappa-1)}\left(\frac{s}{1+s}\right)^{\kappa+1} \frac{\varepsilon}{\sqrt{r}(t-r)^{\kappa-1 / 2}} \min \left\{1, x-\frac{1}{2}\right\}\left(1-\frac{t-r}{t+r}\right)
\end{aligned}
$$




$$
\geq \frac{1}{3 \pi} \min \left\{\frac{2}{2 \varkappa-1}, 1\right\}\left(\frac{s}{1+s}\right)^{\kappa+1} \frac{\varepsilon}{\sqrt{r}(t-r)^{\kappa-1 / 2}} .
$$

Hence we obtain for $(r, t) \in \Sigma^{\prime}$

$$
\overline{u_{0}}(r, t) \geq \begin{cases}\frac{C_{0} \varepsilon}{r^{\kappa}} & \text { if } \quad 0<\varkappa \leq \frac{1}{2}, \\ \frac{C_{0} \varepsilon}{\sqrt{r}(t-r)^{\kappa-1 / 2}} & \text { if } \quad x>\frac{1}{2},\end{cases}
$$

where $C_{0}$ is a constant defined by

(5.3) $\quad C_{0}=\frac{1}{3 \pi} \min \left\{\left|\frac{2}{2 \varkappa-1}\right|, 1\right\}\left(\frac{s}{1+s}\right)^{\kappa+1}$.

For $(r, t) \in \Sigma^{\prime}$ we find that

$$
r^{\kappa} \leq r^{\kappa+1 / 2}(t-r-s)^{-1 / 2}
$$

Therefore it follows (4.20) and (5.2) that

$$
\bar{u}(r, t) \geq\left\{\begin{array}{lll}
\frac{C_{0} \varepsilon(t-r-s)^{1 / 2}}{r^{\kappa+1 / 2}} & \text { if } & 0<\varkappa \leq \frac{1}{2}, \\
\frac{C_{0} \varepsilon}{\sqrt{r}(t-r)^{\kappa-1 / 2}} & \text { if } & x>\frac{1}{2} .
\end{array}\right.
$$

Now, as in the proof of Theorem 2, assume that $\bar{u}$ has more general estimate (4.28). Hence we have (4.29), (4.30) for $(r, t) \in \Sigma^{\prime}$. Using the values

$$
\begin{aligned}
& q=x+\frac{1}{2}, a=\frac{1}{2}, b=0, C=C_{0} \varepsilon \quad \text { if } \quad 0<x \leq \frac{1}{2}, \\
& q=\frac{1}{2}, a=0, b=x-\frac{1}{2}, C=C_{0} \varepsilon \quad \text { if } \quad x>\frac{1}{2}
\end{aligned}
$$

corresponding to (5.4), we obtain (4.29) with

$$
\begin{aligned}
& a^{*}=\frac{p}{2}+2, \quad b^{*}=p x+\frac{p-1}{2}, C^{*}=\frac{D_{\kappa+1 / 2}\left(C_{0} \varepsilon\right)^{p}}{(p / 2+2)^{2}} \text { if } 0<x \leq \frac{1}{2}, \\
& a^{*}=2, \quad b^{*}=p x-\frac{1}{2}, C^{*}=\frac{D_{1 / 2}\left(C_{0} \varepsilon\right)^{p}}{4} \text { if } x>\frac{1}{2}
\end{aligned}
$$

Define the sequence $\left\{a_{\ell}\right\},\left\{b_{\ell}\right\},\left\{C_{\ell}\right\}(\ell \in N)$ by (4.33) in which (4.32) is replaced by (5.6). Solving these sequences we get 


$$
\begin{aligned}
& a_{\ell}=\left(\frac{2}{p-1}+\frac{1}{2}\right) p^{\ell}-\frac{2}{p-1}, b_{\ell}=\left(x+\frac{1}{2}\right) p^{\ell}-\frac{1}{2} \quad \text { if } 0<x \leq \frac{1}{2}, \\
& a_{\ell}=\frac{2}{p-1} p^{\ell}-\frac{2}{p-1}, b_{\ell}=x p^{\ell}-\frac{1}{2} \quad \text { if } \quad x>\frac{1}{2},
\end{aligned}
$$

If $0<x \leq 1 / 2$, then

$$
p a_{\ell}+2=\left(\frac{2}{p-1}+\frac{1}{2}\right) p^{\ell+1}-\frac{2}{p-1} \leq 2(\ell+2) p^{\ell+1} .
$$

If $x>1 / 2$, then

$$
p a_{\ell}+2=\frac{2}{p-1}\left(p^{\ell+1}-1\right) \leq 2(\ell+2) p^{\ell+1} .
$$

Thus, in the same manner as the proof of Theorem 2 , the estimate for $\bar{u}$, (4.37), in which $J$ is replaced by $J^{\prime}$, holds for $(r, t) \in \Sigma^{\prime} . \quad J^{\prime}(r, t)$ is defined by

$$
\begin{aligned}
& J^{\prime}(r, t)=p^{-1} \log C_{1}-S(p)+ \\
& +\left\{\begin{array}{l}
\left(\frac{2}{p-1}+\frac{1}{2}\right) \log (t-r-s)-\left(x+\frac{1}{2}\right) \log (t-r) \text { if } 0<x \leq \frac{1}{2}, \\
\frac{2}{p-1} \log (t-r-s)-x \log (t-r) \text { if } x>\frac{1}{2},
\end{array}\right.
\end{aligned}
$$

where $S(p)$ is the one in (4.36).

$J^{\prime}(r, t)>0$ which lead to the contradiction as in section 4 follows from

$$
\left(\frac{2}{p-1}-x\right) \log (t-r)>\log C^{\prime} \varepsilon^{-1} \text { for }(r, t) \in \Sigma^{\prime} \text { and } t-r \geq 2 s,
$$

where

$$
C^{\prime}=\left((p / 2+2)^{2} D_{1}^{-1}\right)^{1 / p} C_{0}^{-1} e^{S(p)+2 p /(p-1)}
$$

becuase (4.41) is still valid in this section though $C_{0}$ is defined by (5.3). Therefore, setting $t=2 r$, we find that $J^{\prime}(r, t)>0$ for $(r, t) \in \Sigma^{\prime}$ follows from

$$
t>C \varepsilon^{\left(\kappa-\frac{2}{p-1}\right)^{-1}} \text { for } p>1 \text { and } 0<x<\frac{2}{p-1}
$$

where

$$
C=2\left(C^{\prime}\right)^{\left(\kappa-\frac{2}{p-1}\right)^{-1}} .
$$

Thus we conclude that $T$ has to satisfy 


$$
T \leq C \varepsilon^{\left(\kappa-\frac{2}{p-1}\right)^{-1}} .
$$

This completes the proof of Theorem 3.

\section{Appendix}

In Appendix, we give a simple proof of the global existance of solutions to (1.1) with $p>p_{0}(2)$. Following R. T. Glassey [3], we define the norm for a continuous function $u(x, t)$ in $\boldsymbol{R}^{2} \times[0, \infty)$ satisfying (2.1):

(A. 1) $\quad\|u\|=\sup _{(x, t) \in \boldsymbol{R}^{2} \times[0, \infty)} N(|x|, t)|u(x, t)|$,

where

(A. 2) $\left\{\begin{array}{l}k^{-q-1 / 2}(t+r+2 k)^{1 / 2}(t-r+2 k)^{q} \text { for } p>p_{0}(2), p \neq 4 \\ k^{-1}(t+r+2 k)^{1 / 2}(t-r+2 k)^{1 / 2}\left(\log \frac{t-r+3 k}{k}\right)^{-1} \text { for } p=4\end{array}\right.$

and

$$
\begin{aligned}
& q=\frac{p-3}{2} \quad \text { for } \quad p_{0}(2)<p<4 \\
& q=\frac{1}{2} \quad \text { for } \quad p>4
\end{aligned}
$$

We notice that the norm is slightly modified the one in [3].

We shall show the following lemma which assures, by the method in $\S 2$, the global in time existence of solutions to (1.1) with small initial data.

LEMMA A.1. There exists a positive constant $C$ depending only on $p$ such that

(A. 4) $\left\|L\left(|u|^{p}\right)\right\| \leq C k^{2}\|u\|^{p}, p>p_{0}(2)$,

for any continutus function $u$ in $\boldsymbol{R}^{2} \times[0, \infty)$ satisfying (2.1).

PROOF: The proof will be done by same method as in $\S 3$. In order to show the a priori estimate (A.4), it is enough to prove, instead of (3. 7),

(A. 5) $\quad I_{j}(r, t) \leq C k^{2} N(r, t)^{-1}$ for $j=1,2$,

where the $I_{j}$ are defined in (3.6).

We first treat the integral $I_{1}$. When $4 r>t+r+2 k$, we get, instead of (3. 13), 
(A. 6)

$$
I_{1}(r, t) \leq \frac{\pi k^{p q+p / 2}}{8 \sqrt{2 r}} \int_{t-r}^{t+r} \frac{(\alpha+2 k)^{(2-p) / 2}}{\sqrt{\alpha-(t-r)}} d \alpha \int_{-k}^{t-r}(\beta+2 k)^{-p q} d \beta
$$

The $\beta$-integral for $p=4$ is

(A. 7)

$$
\int_{-k}^{t-r}(\beta+2 k)^{-2}\left(\log \frac{\beta+3 k}{k}\right)^{4} d \beta
$$

Since $p>p_{0}(2)>3$, it follows from (3. 14) that the $\alpha$-integral in (A.6) is dominated by

$$
2\left(\sqrt{2}+\frac{p-2}{p-3}\right)(t-r+2 k)^{(3-p) / 2} .
$$

Note that $p q>1$ for $p>p_{0}$ (2). Then the $\beta$-integral in (A.6) is dominated by

$$
\int_{-k}^{\infty}(\beta+2 k)^{-p q} d \beta=\frac{k^{1-p q}}{p q-1} .
$$

The $\beta$-integral (A. 7) is also dominated by

$$
C k^{-\delta} \int_{-k}^{\infty}(\beta+2 k)^{-2+\delta} d \beta=\frac{C k^{-1}}{1-\delta}
$$

for some small $\delta>0$ and for some $C>0$. Hence we get

(A. 8) $\quad I_{1}(r, t) \leq C(p) k^{1+p / 2}(t+r+2 k)^{-1 / 2}(t-r+2 k)^{(3-p) / 2}$.

Since

$$
\begin{aligned}
& (t-r+2 k)^{(4-p) / 2} \leq k^{(4-p) / 2} \quad \text { for } \quad p>4, \\
& \log \frac{t-r+3 k}{k}>\log 2 \quad \text { for } \quad p=4,
\end{aligned}
$$

we conclude from (A. 8) that (A. 5) for $I_{1}$ is valid.

When $4 r<t+r+2 k$, we get, instead of (3.16)

$$
\begin{gathered}
\text { (A. 9) } \quad I_{1}(r, t) \leq \frac{\pi}{8} k^{p q+p / 2} \int_{t-r}^{t+r} \frac{(\alpha+2 k)^{(1-p) / 2}}{\sqrt{\alpha-(t-r)}} d \alpha \int_{-k}^{t-r}(\beta+2 k)^{-p q} d \beta \\
\text { for } \quad p \neq 4
\end{gathered}
$$

The $\beta$-integral becomes (A. 7) for $p=4$. Since $p>p_{0}(2)>2$ and $(t+r+2 k)$ is equivalent to $(t-r+2 k)$, it follows from (3.17) that the $a$-integral in (A. 9) is dominated by

$$
C(p)(t+r+2 k)^{-1 / 2}(t-r+2 k)^{(3-p) / 2} .
$$


Hence we also conclude in the same way as above that (A.5) for $I_{1}$ is valid.

Next we treat the integral $I_{2}$. Making use of the method deriving (3. 21 ), we find that the $\alpha$-integral in (3.19) or (3.20) is dominated by

$$
C(p)(t-r+2 k)^{(3-p) / 2} \text { for } p_{0}(2)<p<4,
$$

(A. 10) $C(p)(t-r+2 k)^{-1 / 2} \log \frac{t-r+3 k}{k}$ for $p=4$,

$$
C(p) k^{(4-p) / 2}(t-r+2 k)^{-1 / 2} \text { for } p>4 .
$$

When $4 r>t+r+2 k$, we get, instead of (3. 19),

$$
\begin{gathered}
\text { (A. 11) } I_{2}(r, t) \leq \frac{\pi k^{p q+p / 2}}{8 \sqrt{2 r}} \int_{0}^{t-r} \frac{(\alpha+2 k)^{(2-p) / 2}}{\sqrt{t-r-\alpha}} d \alpha \int_{-k}^{t-r}(\beta+2 k)^{-p q} d \beta \\
\text { for } p \neq 4 .
\end{gathered}
$$

The $\beta$-integral becomes (A. 7) for $p=4$. Since the $\beta$-integral in (A. 11) is dominated by $C(p) k^{1-p q}$, we find from (A. 10) and (A. 11) that (A. 5) for $I_{2}$ is valid.

When $4 r<t+r+2 k$, i.e., $t+r+2 k<2(t-r+2 k)$, we get, instead of (3.20),

$$
\text { (A. 12) } \quad I_{2}(r, t) \leq \frac{\pi}{8} k^{p q+p / 2} \int_{0}^{t-r} \frac{(\alpha+2 k)^{(2-p) / 2}}{\sqrt{t-r-\alpha}} d \alpha \int_{-k}^{t-r} \frac{(\beta+2 k)^{-p q}}{\sqrt{t-r-\beta}} d \beta
$$

The $\beta$-integral for $p=4$ is

(A. 13) $\int_{-k}^{t-r} \frac{(\beta+2 k)^{-2}}{\sqrt{t-r-\beta}}\left(\log \frac{\beta+3 k}{k}\right)^{4} d \beta$.

Making use of the method deriving (3.22), we find that the $\beta$-integrals are dominated by

(A. 14) $C(p) k^{1-p q}(t+r+2 k)^{-1 / 2}$.

We give here the proof of (A. 14) for the critical case where $p=4$ and $t$ $-r>k$, which implies $t-r>(t-r+2 k) / 4$. We find that, for some $C>0$ and some small $\delta>0$, the integral (A. 13) is dominated by

$$
C k^{-\delta} \int_{-k}^{t-k} \frac{(\beta+2 k)^{-2+\delta}}{\sqrt{t-\gamma-\beta}} d \beta
$$

Then, breaking the integral up into two pieces, we get 


$$
\begin{aligned}
C k^{-\delta} \int_{-k}^{(t-r) / 2} \frac{(\beta+2 k)^{-2+\delta}}{\sqrt{t-r-\beta}} d \beta & \leq \sqrt{2} C k^{-\delta}(t-r)^{-1 / 2} \int_{-k}^{\infty}(\beta+2 k)^{-2+\delta} d \beta \\
& \leq \frac{2 \sqrt{2} C k^{-1}}{1-\delta}(t-r+2 k)^{-1 / 2} \\
& \leq \frac{4 C k^{-1}}{1-\delta}(t+r+2 k)^{-1 / 2}
\end{aligned}
$$

On the complement,

$$
\begin{aligned}
C k^{-\delta} \int_{(t-r) / 2}^{t-r} \frac{(\beta+2 k)^{-2+\delta}}{\sqrt{t-r-\beta}} d \beta & \leq C k^{-\delta}\left(\frac{t-r}{2}+2 k\right)^{-2+\delta} \int_{(t-r) / 2}^{t-r} \frac{d \beta}{\sqrt{t-r-\beta}} \\
& \leq C k^{-\delta} 2^{2-\delta}(t-r+2 k)^{\delta-3 / 2} . \\
& \leq C \sqrt{2} 2^{2-\delta} k^{-1}(t+r+2 k)^{-1 / 2} .
\end{aligned}
$$

Here we have used that $(t-r+2 k)^{\delta-1} \leq k^{\delta-1}$. Therefore, we conclude from (A. 10), (A. 12) and (A. 14) that (A. 5) for $I_{2}$ is valid. Thus the proof of Lemma A. 1 is completed.

\section{References}

[1] R. AGEMI, Blow-up of solutions to nonlinear wave equations in two space dimensions, Manuscripta Math. 73 (1991), 153-162.

[2] F. ASAKURA, Existence of a global solution to a semi-linear wave equation with slowly decreasing initial data in three space dimensions, Comm. in PDE 11(13) (1986), 1459-1487.

[ 3 ] R. T. GLASSEY, Existence in the large for $\square u=F(u)$ in two space dimensions, Math. $Z$. 178 (1981), 233-261.

[4] R.T. GLASSEY, Finite-time blow-up for solutions of nonlinear wave equations, Math. Z. 177 (1981), 323-340.

[5] F. JOHN, Plane waves and spherical means applied to partial differential equations, Interscience (1955), New York.

[6] F. JOHN, Blow-up of solutions of nonlinear wave equations in three space dimensions, Manuscripta Math. 18 (1979), 235-268.

[7] F. JOHN, Nonlinear wave equations, Formation of singularities, Pitcher Lectures in the mathematical sciences, Lehigh University, University Lecture Series, American Math. Soc. Providence (1990).

[8] K. KUBOTA, Existence of a global solution to a semi-linear wave equation with initial data of non-compact support in low space dimensions, Hokkaido Univ. Preprint Ser, Math. \#129 (1991).

[9] H. LindBlAD, Blow-up for solutions of $\square u=|u|^{p}$ with small data, Comm. in PDE 15(6) (1990), 757-821.

[10] J. SCHAEFFER, The equation $u_{t t}-\Delta u=|u|^{p}$ for the critical value of $p$, Proc. Roy, Soc. Edinburgh, 101 A (1985), 31-44.

[11] K. TSUTAYA, Global existence theorem for semilinear wave equations with non compact data in two space dimensions, to appear in J. Differential Equations. 
Department of Mathematics

Hokkaido University

060 Sapporo, Japan

Current address of the second auther Institute of Mathematics

University of Tsukuba

305 Tsukuba, Japan 